\title{
Incidência de escoliose idiopática em uma escola pública do município de Suzano/SP
}

Idiopathic scoliosis incidence in

public school in Suzano town

FisiSenectus . Unochapecó Ano 4, n. 2 - Jul/Dez. 2016 p. $3-11$

Marcella Lopes de Proença. marcellaproenca@hotmail.com

Acadêmica do Curso de Fisioterapia da Universidade Braz Cubas - UBC - Mogi das Cruzes - SP.

Gabriela dos Santos Assis. gabrielafisio@outlook.com.br

Acadêmica do Curso de Fisioterapia da Universidade Braz Cubas - UBC - Mogi das Cruzes - SP.

Carlos Alberto dos Santos. fisiocarlos@globo.com

Fisioterapeuta. Mestre em Ortopedia - UNIFESP. Prof. do Curso de Fisioterapia da Universidade Braz Cubas - UBC - Mogi das Cruzes - SP.

Laila Moussa. IImoussa@hotmail.com

Fisioterapeuta. Mestre em Ciências do Movimento - UNG. Prof. a do Curso de Fisioterapia da Universidade Braz Cubas - UBC - Mogi das Cruzes - SP.

Hebert Ailton Stefano da Silva. hebert@fisioterapiasuzano.com.br

Fisioterapeuta. Especialista em Fisioterapia Cardiorrespiratória, Ortopedia e Musculoesquelético e Acupuntura.

\section{Resumo}

Introdução: 0 termo escoliose define-se como desvio lateral do plano frontal do corpo, associado ou não à rotação dos corpos vertebrais. Na infância iniciam-se os sinais da escoliose, que pode evoluir durante o crescimento e se instalar se não tratada. A fisioterapia ainda é pouco atuante no campo escolar, o que denota a necessidade de maior atenção. Objetivo: Avaliar a incidência de escoliose em crianças de uma escola Pública do Município de Suzano. Materiais e Métodos: Participaram do estudo crianças dos 6 aos 11 anos, ambos os Gêneros, matriculados em uma escola Pública do Município de Suzano. Uma palestra de orientação, foi ministrada para os responsáveis. Realizou-se uma avaliação postural por meio de uma ficha, contendo parâmetros relacionados à escoliose, uma Escala Facial de Dor e o teste de Adams. Os resultados foram tabulados através de dados estatísticos percentuais em planilha do programa Microsoft ${ }^{\circledR}$ Office Excel 2013. Resultados: Os resultados demonstram que $16,67 \%$ dos alunos avaliados tiveram teste de Adams positivo, apresentaram escoliose os alunos de todas as idades, prevalência no gênero masculino. Constatou-se que, das crianças com assimetrias posturais, 16,82\% estavam relacionadas com escoliose e $57,94 \%$ possuíam assimetrias posturais sem escoliose. Considerações Finais: De acordo com os resultados encontrados, pode-se afirmar a importância da avaliação postural em escolares para um diagnóstico precoce. 0 presente estudo realizou

\section{Fisißenectus}


avaliação durante o período escolar, sem equipamentos de alto custo, tendo como principal método o teste de Adams e uma ficha de avaliação postural, comprovando ser possível a implantação da fisioterapia nas escolas, evitando assim, diagnósticos tardios.

\title{
Palavras-chave
}

Incidência; Escoliose; Criança.

\begin{abstract}
Introduction: The term Scoliosis is defined as lateral deviation of the body of the frontal plane, with or without rotation of the vertebral bodies. In the childhood the signs of scoliosis begins, what may develop during growth and settle if left untreated. Physical therapy is still not very active in the school field, which indicates the need for greater attention. Objective: Evaluate the incidence of scoliosis in the children from a public school in Suzano. Materials and methods: Children aged 6 to 11 participated of the study, both genders, enrolled in a public school in Suzano town. An orientation lecture was given to the responsible ones. It was held a postural assessment through a form containing parameters related to scoliosis, a facial scale and the Adams'test. The results were tabulated by percentage statistical data in the Microsoft Office Excel 2013 spreadsheet program. Results: The results showed that $16,67 \%$ of students of all ages, prevailed in males. It was found that children with postural asymmetry $16,82 \%$ related to scoliosis and $57,94 \%$ dad postural asymmetry without scoliosis. Conclusions: According to the results it can be stated the importance of postural evaluation in school for an early diagnosis. This study conducted the evaluation during school period without costly equipment, the main method used was the Adams' test, and a postural assessment form, proving possible the implantation of physiotherapy in schools, thus avoiding late diagnosis.
\end{abstract}

\section{Keywords}

Incidence; Scoliosis; Children.

\section{Introdução}

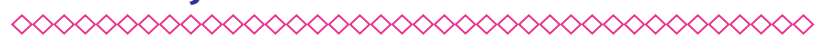

O termo escoliose tem origem grega e significa curvatura; portanto, é definido como desvio lateral do plano frontal do corpo, associado ou não à rotação dos corpos vertebrais nos planos axial, coronal e sagital ${ }^{1-2}$.

Geralmente, a escoliose pode ocorrer como resultado de uma malformação vertebral congênita, a partir de anomalias na medula espinhal (tumor ou siringomielia), uma desordem neuromuscular, facetária ou degeneração do disco, neurofibromatose, doença do tecido conjuntivo, displasias esqueléticas, iatrogênia, ou apresentar uma causa desconhecida ${ }^{3}$.

Se a coluna é curvada e torcida na região torácica, as costelas são puxadas para fora da posição causando uma protuberância na parte superior das costas. Já a curvatura na coluna vertebral lombar faz com que haja uma protuberância na parte inferior das $\operatorname{costas}^{1}$. Na grande maioria dos casos, a causa específica não é encontrada, e a etiologia é denominada de idiopática. Seu desenvolvimento pode ocorrer desde a infância e agravar-se na adolescência².

A escoliose idiopática pode ser classificada de acordo com a idade de início como infantil, juvenil ou adolescente. Em experiência clínica diária, é a idade de diagnóstico, e não de início, que orientam essa classificação, por isso, se o diagnóstico é feito antes dos 4 anos, a escoliose é definida como "infantil", se o diagnóstico vem a partir de 4 a 9 anos de idade, chamamos de escoliose idiopática "juvenil", e depois dos 10 anos de idade a escoliose idiopática é classificada como do "adolescente"4.

A forma de escoliose idiopática juvenil é geralmente considerada como mais provável para o progresso e menos propensa a responder a órtese e com mais chance de exigir intervenção cirúrgica ${ }^{4}$.

No caso da escoliose idiopática do adolescente, o momento de maior risco para a progressão da doença ocorre no período da puberdade, em que o crescimento ósseo ocorre de forma muito rápida, havendo, portanto, a necessidade de meios de 
diagnóstico precoce e de intervenção eficaz para evitar a progressão da doença².

A alta incidência de desvios posturais na infância, estimada em $20 \%$, além de preocupante, é um problema de saúde pública. Na infância, iniciam-se os sinais da escoliose, que podem evoluir durante o crescimento. E esse problema pode se instalar definitivamente se não for tratado ${ }^{5}$.

A progressão da curva é relacionada a vários fatores: o padrão e a magnitude da curva, a idade do paciente no momento da apresentação, o sinal de Risser e o estado de menarca do paciente ${ }^{4}$.

No estirão de crescimento, entre 7 e 10 anos, há potencial evolutivo da atitude escoliótica, pois a criança está sujeita à alteração de postura na vida cotidiana e já está em fase escolar ${ }^{5}$.

A avaliação em escola pode ser usada para identificar crianças que podem ter escoliose, bem como aquelas que podem estar em alto risco para a doença. Alguns países desenvolvidos já adotam a realização sistemática de avaliações posturais durante a fase escolar para identificar e acompanhar a progressão das alterações da postura em geral e, principalmente, da postura da coluna vertebral. Desse modo, as avaliações posturais, em que os indivíduos são submetidos a testes não invasivos, tornam-se opção viável para estudos das alterações da postura corporal. Sob o ponto de vista ortopédico, as avaliações posturais em escolares são benéficas e proporcionam oportunidade individual de diagnóstico precoce ${ }^{6}$.

OSchool Screening, um programa recomendado pela Sociedade de Pesquisa em Escoliose e pela Academia Americana de Cirurgiões Ortopédicos e obrigatório por lei em 26 estados dos Estados Unidos, é uma das opções para avaliação da coluna vertebral de indivíduos em idade escolar. Trata-se de mensuração clínica da escoliose que identifica as curvas em estágio precoce, por isso assintomático, de desenvolvimento. Esse programa, além de reduzir a demanda de intervenções cirúrgicas, diminui os gastos financeiros relacionados ao tratamento da enfermidade ${ }^{7}$.

A fisioterapia ainda é pouco atuante no campo escolar, o que denota a necessidade de maior atenção, especialmente em relação aos aspectos preventivos posturais. No Brasil, os programas de rastreamento escolar da escoliose não são tão difundidos e pouco se sabe sobre o real estado em que se encontram as colunas vertebrais dos escolares brasileiros. Diante das evidências apresentadas, destaca-se a relevância científica e social de se investigar a escoliose ${ }^{6,7}$.

0 objetivo desta pesquisa foi embasado na referida necessidade de verificação precoce. Para identificação da patologia ou potenciais riscos de seu desenvolvimento, foi realizado um trabalho de campo, em crianças de uma escola do município de Suzano, inicialmente através de métodos não invasivos, com o intuito de obter um diagnóstico e fazer uma intervenção precoce em crianças com escoliose.

\section{Materiais e métodos}

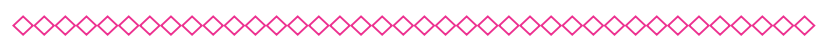

O trabalho foi realizado mediante a aprovação do Comitê de Ética da Universidade Braz Cubas, sob o parecer $n^{\circ} 026 / 15$. Poderiam participar do estudo 673 crianças dos 6 aos 11 anos, dos gêneros feminino e masculino, matriculados no período da manhã da escola EMEF Antônio Marques Figueira, localizada no Município de Suzano/SP, desde que devidamente autorizadas pelos seus responsáveis. Inicialmente, uma palestra de orientação sobre a escoliose foi ministrada para pais e responsáveis que compareceram à reunião bimestral, onde foi apresentado o Termo de Consentimento Livre e Esclarecido e assinaram os que concordaram em participar da pesquisa.

Foram incluídas no estudo crianças de 6 a 11 anos, dos gêneros feminino e masculino, matriculadas na EMEF Antônio Marques Figueira, devidamente autorizadas pelos seus responsáveis. Foi realizada uma avaliação postural por meio de uma ficha contendo parâmetros relacionados à escoliose que incluem posturas na visão anterior, lateral e posterior, nos dias 13 e 20 de outubro.

Uma Escala Facial de Dor: a escala FPS para adultos e crianças também é uma escala de categorias, mas com descritores visuais, usando, no caso, expressões faciais que refletem magnitudes de intensidade e dor diferentes. As crianças selecionaram a face que consistia com seu nível atual de dor. No caso do presente estudo, empregaram-se números de 0 a 5 para facilitar a tabulação dos dados ${ }^{8}$. 
Foi realizado, também, o teste de Adams, sendo seguidas as recomendações clássicas: observador colocado em posição posterior, participante em flexão anterior do tronco com os membros superiores para a frente até ao ponto máximo de alcance, superfícies palmares das mãos unidas, pés juntos e joelhos estendidos. 0 teste foi considerado positivo se, na visão tangencial do dorso, existisse visualização de gibosidade, desnível e/ou existência de curvaturas toracolombares ${ }^{9}$.

Os testes foram realizados na própria escola pelas autoras deste trabalho e três voluntárias estudantes do Curso de Fisioterapia da Universidade Braz Cubas. Os alunos foram avaliados de forma individual: os meninos retiraram a camisa para facilitar a visualização de possíveis desvios posturais, e as meninas foram avaliadas com as mamas devidamente cobertas, facilitando a visualização dos desvios e impedindo possíveis constrangimentos. Após a avaliação, os resultados foram tabulados através de dados estatísticos percentuais em planilha do programa Microsoft ${ }^{\circledR}$ Office Excel 2013.

\section{Resultados}

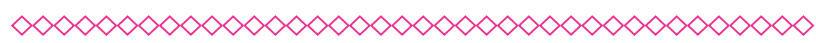

A amostra poderia ter sido composta por 673 voluntários, porém muitos pais não compareceram à reunião e, entre os que compareceram, 107 autorizaram a participação das crianças no presente estudo, sendo 63 (58,88\%) do gênero feminino e $44(41,12 \%)$ do gênero masculino, com idades entre 6 e 11 anos, sendo 20 (18,69\%) com 6 anos, 51 (47,66\%) com 7 anos, 12 (11,21\%) com 8 anos, 9 (8,41\%) com 9 anos, 10 (9,35\%) com 10 anos e 5 $(4,67 \%)$ com 11 anos. Do total das crianças participantes, $18(16,67 \%)$ apresentaram teste positivo para Escoliose (Gráfico 1).

Os Gráficos 2 e 3 demonstram a correlação entre a idade e a presença de escoliose e a correlação entre os gêneros e a escoliose respectivamente. Observou-se que apresentaram escoliose, as crianças de todas as idades, sendo $2(1,87 \%)$ com 6 anos, $9(8,41 \%)$ com 7 anos, $3(2,80 \%)$ com 8 anos, $1(0,93 \%)$ com 9 anos, 2 (1,87\%) com 10 anos, 1 (0,93\%) com 11 anos. Na relação entre os gêneros e a escoliose, constatou-se que apresentaram escoliose $9(8,41 \%)$ crianças do gênero feminino e
9 (8,41\%) do gênero masculino. 54 (50,47\%) do gênero feminino e 35 (32,71\%) do gênero masculino não apresentaram escoliose.

O Gráfico 4 descreve a correlação entre os tipos de escoliose. Observou-se que $9(8,41 \%)$ das crianças apresentaram escoliose torácica à direita, em $8(7,48 \%)$ constatou-se escoliose torácica à esquerda e $1(0,93 \%)$ apresentou escoliose toracolombar à direita. Entre as 18 crianças que apresentaram teste de Adams positivo, $8(44,44 \%)$ apresentaram dor na Escala Facial de Dor e 10 (55,56\%) negaram sentir dores nas costas (Gráfico 6).

\section{Discussão}

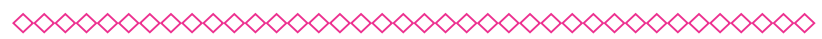

Durante o crescimento, as proporções do corpo atingem gradualmente a forma do adulto. O crescimento é maior no início da infância, diminui até o começo da adolescência e volta a acelerar durante esse período. Apesar da desaceleração na velocidade de crescimento ósseo com os anos, vários aspectos relacionados às posturas e aos hábitos das crianças passam a ser determinantes para o desenvolvimento muscular e esquelético. Principalmente no período de 7 a 12 anos de idade, quando ocorre a busca do equilíbrio às novas proporções do corpo, evidenciam-se as transformações posturais ${ }^{10}$.

Araújo et al. ${ }^{11}$, com base na Lei $n^{0}$ 10.759/98 que determina que o peso de mochilas, pastas e similares, mencionam que estes materiais não devem ultrapassar $5 \%$ do peso de crianças da préescola e $10 \%$ do peso do aluno do Ensino Fundamental, percentual indicado pela Organização Mundial da Saúde (OMS), e analisaram a incidência de escoliose com excesso de carga nas mochilas em crianças de 6 a 10 anos. Esses autores verificaram que, em relação ao teste de Adams, de 109 crianças avaliadas, 53 crianças $(48,6 \%)$ tiveram o resultado positivo. Além disso, $26(23,9 \%)$ delas carregavam excesso de carga, o que sugere que os resultados obtidos pelas autoras desta pesquisa possam estar relacionados com o excesso de peso carregado.

Em estudo avaliou crianças pré-escolares de 5 e 6 anos e utilizou, após o teste de Adams positivo, a fotogrametria, para determinar a curvatura. 
Como resultado da amostra, de 377 crianças, 99 possuíam escoliose ${ }^{5}$. 0 presente estudo utilizou o teste de Adams como ferramenta de mensuração da gibosidade, pois, segundo Ferreira et al. ${ }^{7}$, trata-se da base de avaliação em escolares em todo o mundo. A realização de exames radiográficos é o padrão-ouro para o diagnóstico e avaliação da escoliose. Isso expõe tal população a riscos de saúde como alterações genéticas e predisposição ao câncer, devido ao efeito cumulativo da radioatividade no organismo. Por esse motivo, métodos não invasivos têm sido bastante estudados, já que são alternativas para acompanhar a evolução das escolioses.

Quanto ao gênero, a prevalência encontrada na literatura aponta que é maior em meninas, e diferiu do presente achado de maior prevalência em meninos. Concordando com o estudo de Vieira et al. ${ }^{5}$, que teve como resultado a incidência maior no gênero masculino.

Nos resultados, foi constatado que $74,77 \%$ das crianças possuíam alterações posturais, mostrando existir uma relação com o estudo de Meireles et al. ${ }^{12}$, autores que apontaram que assentos que deixam de oferecer uma melhor angulação para o posicionamento dos alunos têm sido compreendidos como um dos indicadores para os problemas posturais de escolares. Foram avaliados, em seu estudo, 2 grupos com diferentes mobiliários, e obtiveram como resultado que, da amostra de 60 alunos, $92 \%$ possuíam desvios posturais.

A dor é subjetiva e envolve mecanismos físicos, psíquicos e culturais que variam de indivíduo para indivíduo, compreendendo aspectos sociais de sua vida ${ }^{13}$. Com abordagem à relação entre dor e escoliose, a literatura é escassa. 0 atual estudo demostrou que há a presença de dor, mas não necessariamente é um sintoma associado ao desvio, concordando com o estudo de Araújo et al. ${ }^{9}$, em que, com relação à dor, o fator escoliose e o excesso de carga não tiveram resultados significantes nesta faixa etária.

\section{Conclusão}

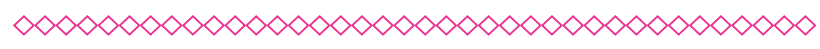

Entre as 107 crianças participantes, 18 (16,82\%) apresentaram teste de Adams positivo, afırmando a importância da avaliação postural em escolares para o diagnóstico precoce, principalmente durante o "estirão" de crescimento, a partir dos 6 anos. 0 presente estudo realizou avaliação nas crianças durante o período escolar, sem equipamentos e materiais de alto custo, tendo como principal método o teste de Adams e uma ficha de avaliação postural, comprovando ser possível e acessível de ser implantado, nas escolas, o profissional fisioterapeuta, a fim de evitar a progressão da escoliose devido a diagnósticos tardios.

\section{Referências}

$\infty \times \infty \times \infty \times \infty \times \infty \times \infty \times \infty \times \infty \times \infty \times \infty \times \infty \times \infty \times \infty)$

1. Honeyman C. Raising awareness of scoliosis among children's nurses. Nursing Children and Young People. 2014; 26(5):30-7.

2. Junior JVS, Sampaio RMM, Aguiar JB, Pinto FJM. Perfil dos desvios posturais da coluna vertebral em adolescentes de escolas públicas do município de Juazeiro do Norte - CE. Fisioter Pesq. 2011; 18(4):311-6.

3. Silva EG, Kojo HD, Joaquim AF, Shaffrey CI. Scoliosis: current overview and treatment considerations. Arq Bras Neurocir. 2010; 29(4):130-6.

4. Donzelli S, Zaina F, Lusini M, Minnella S, Negrini S. In favour of the definition "adolescents with idiopathic scoliosis": juvenile and adolescent idiopathic scoliosis braced after ten years of age, do not show different end results. SOSORT award winner 2014. Scoliosis journal. 2014; 9(7):1-6.

5. Vieira DBALP, Beresoski CM, Camargo MZ, Fernandes KBP, Claudia Siqueira PCM, Fujisawa DS. Sinais precoces de escoliose em crianças préescolares. Fisioter Pesq. 2015; 22(1):69-75.

6. Suh SW, Modi HN, Yang JH, Hong JY. Idiopathic scoliosis in Korean schoolchildren: a prospective screening study of over 1 million children. Eur Spine J. 2011; 20:1087-94.

7. Ferreira DMA, Suguikawa TR, Pachioni CAS, Fregonesi CEPT, Camargo MR. Rastreamento escolar da escoliose: medida para o diagnóstico precoce. Rev Bras Cresc Desen Hum. 2009; 19(3):357-68. 
8. Minson FP, Morete MC, Marangoni MA, Waksman RD, Farah OGD. Manuais de Especialização: Dor. 1. ed. Barueri: Manole; 2015. p. 51-52.

9. Preto LSR, Santos ARR, Rodrigues VMCP, Quitério N F N, Pimentel MH, Manrique GA. Análise por Fotogrametria da Postura e Fatores de Risco Associados em Crianças e Adolescentes Escolarizados. Rev Enf. 2015; 7:31-40.

10. Santos CIS, Cunha ABN, Braga VP, Saad IAB, Ribeiro MAGO, Conti PBM, et al. Ocorrência de desvios posturais em escolares do ensino público fundamental de Jaguariúna, São Paulo. Rev Paul Pediatr. 2009; 27(1):74-80.
11. Araújo AGS, Guimbala AL, Cidral SIA, Woellne SS. Incidência de escoliose com excesso de carga nas mochilas em crianças de 6 a 10 anos. Rev Bras Fis Exercício. 2012; 11(2):105-10.

12. Meireles HR, Junior JHAF, Junior JEGL, Figueiredo ADJ. Influência das Carteiras Escolares na Postura de Alunos da Rede Pública do Município de Cajazeiras-PB. Rev Fisioter S Fun. 2013; 2(1):35-41.

13. Rossato $L M$, Ebner $C$, Nascimento $L C$, Damião EBC, Rocha MCP, Guedes DMB, et al. Facilidades e dificuldades identificadas pelas enfermeiras pediatras na aplicação dos "cartões de qualidade da dor". Rev Saúde. 2015; 15(40):3-14. 


\section{Anexos}

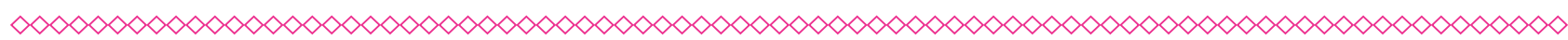

Gráfico 1 - Total de crianças que apresentaram Teste de Adams positivo ou negativo - 2016.

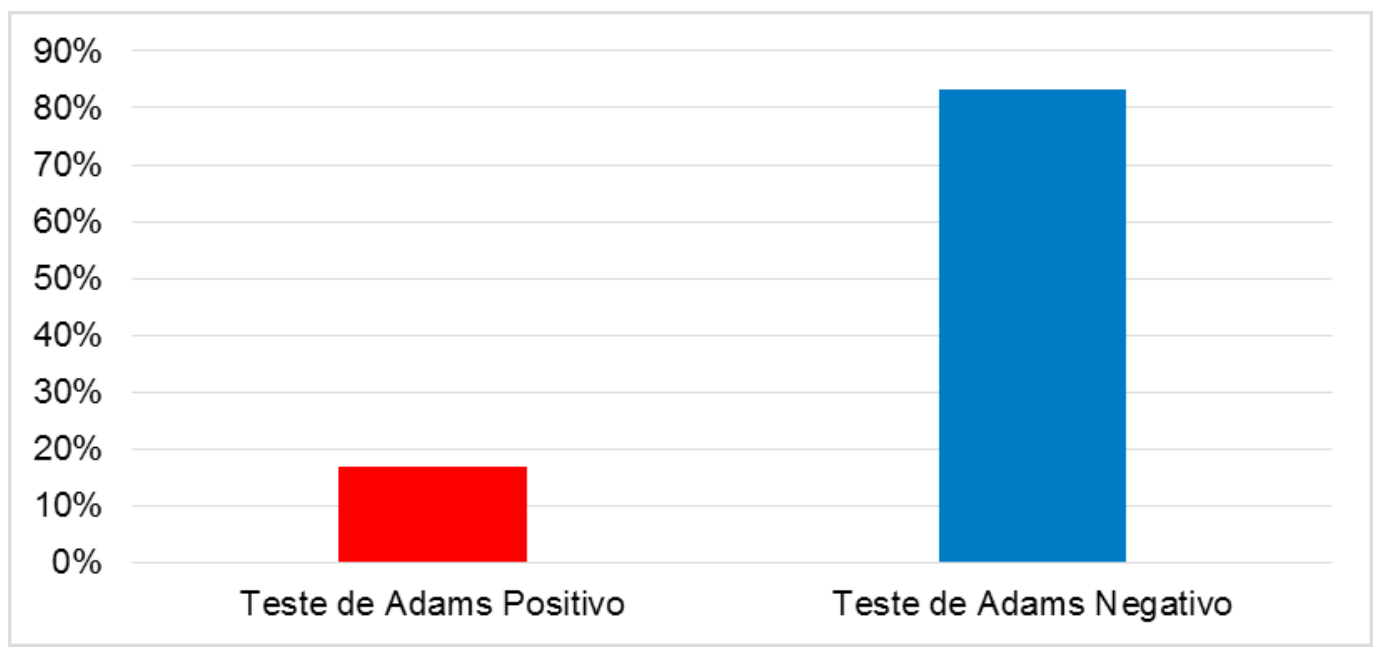

(clique para voltar ao texto)

Gráfico 2 - Correlação entre idades e presença de Escoliose - 2016.

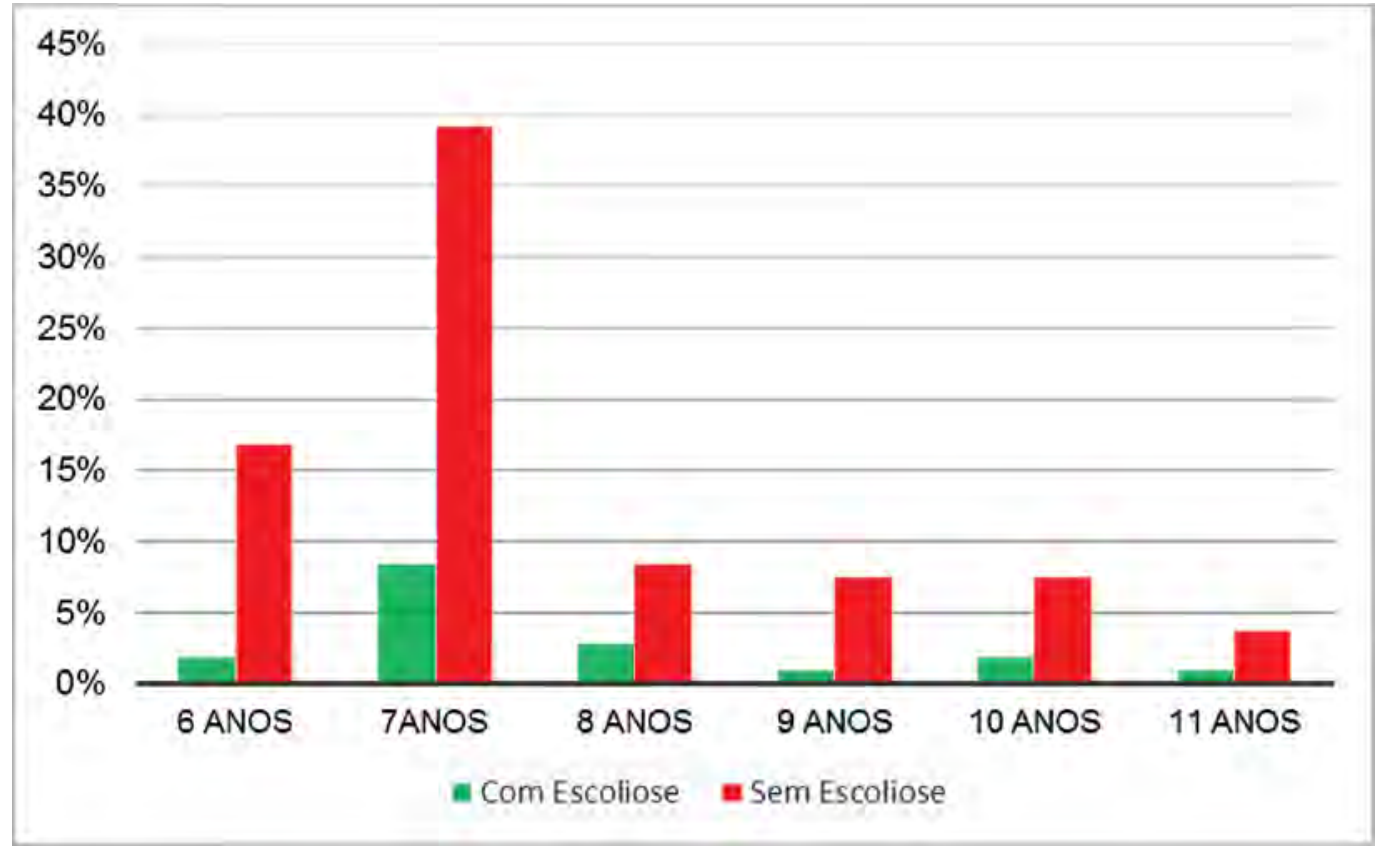

(clique para voltar ao texto) 
Gráfico 3 - Correlação entre gêneros e escoliose - 2016.

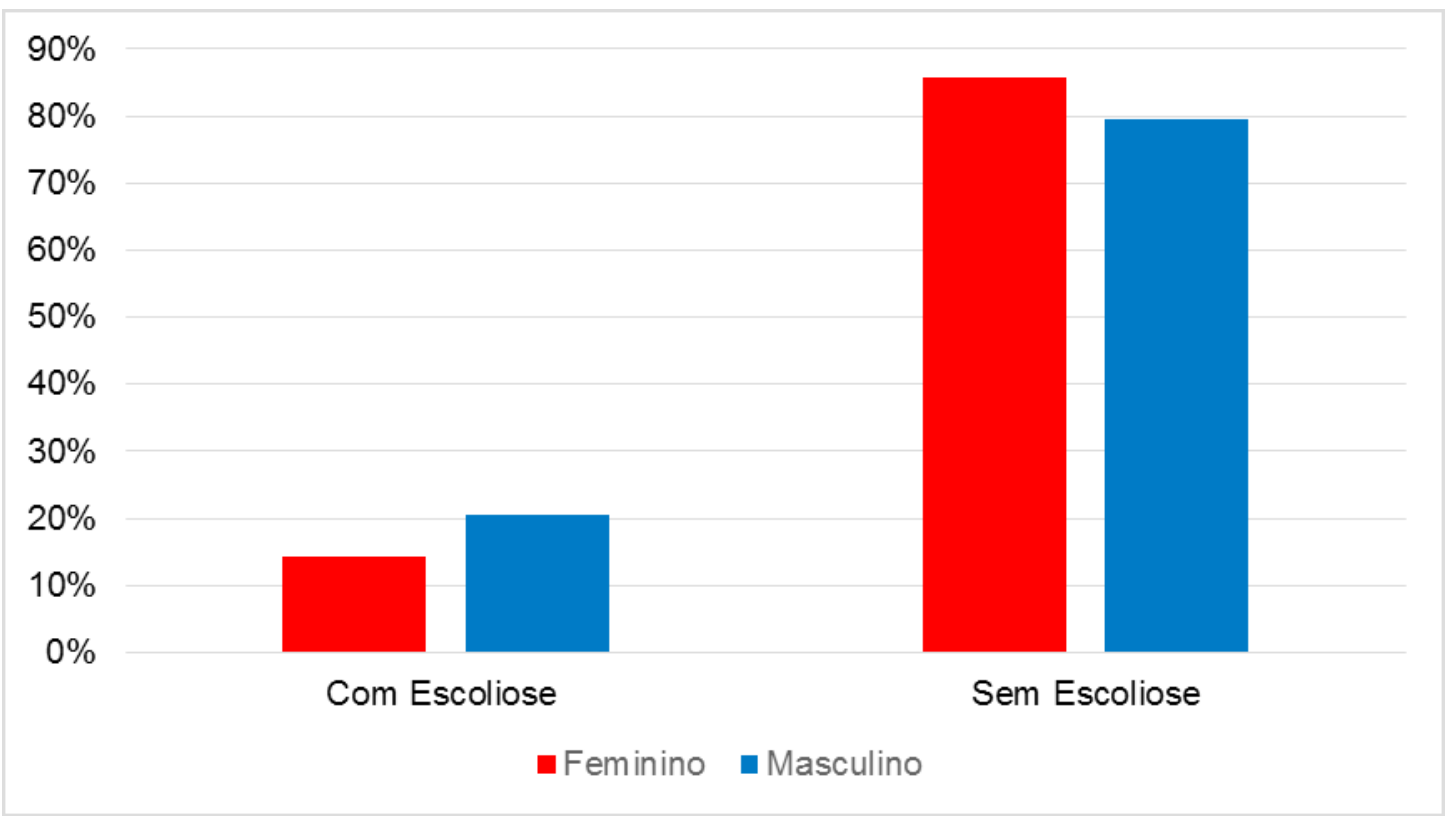

(clique para voltar ao texto)

Gráfico 4 - Correlação entre os tipos de escoliose - 2016.

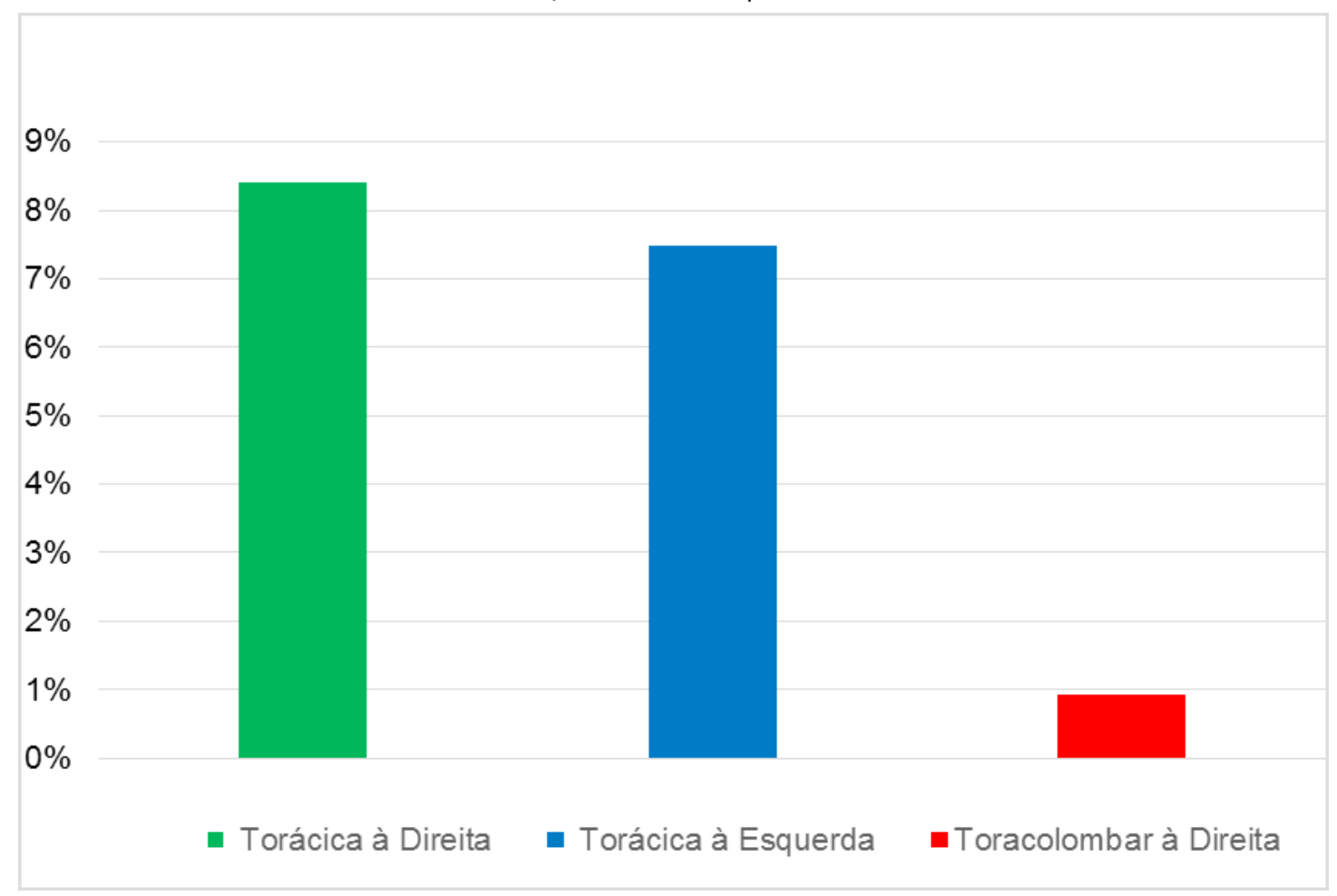

(clique para voltar ao texto) 
Gráfico 5 - Correlação entre assimetrias posturais e a presença ou não de escoliose - 2016.

$70 \%$

$60 \%$

$50 \%$

$40 \%$

$30 \%$

$20 \%$

$10 \%$

$0 \%$
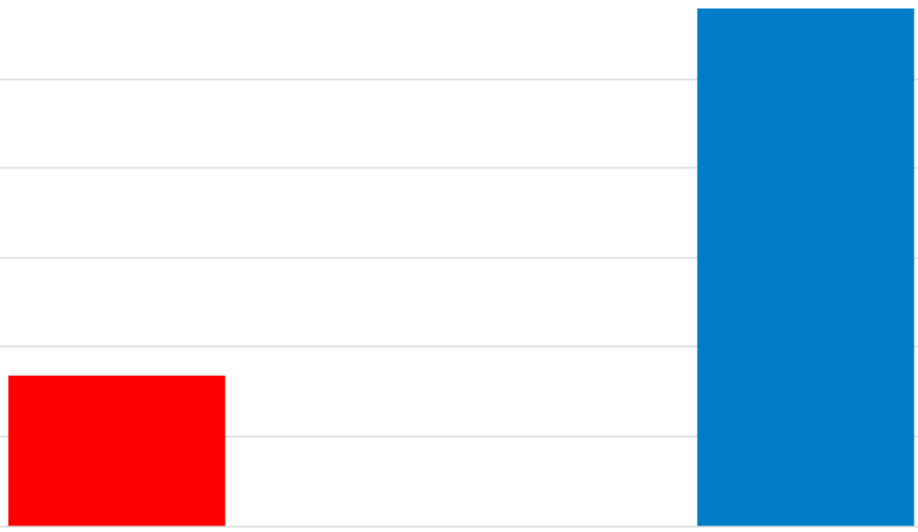

- Com Escoliose $\quad$ Sem Escoliose

(clique para voltar ao texto)

Gráfico 6 - Dor e ausência de dor entre as crianças com teste de Adams positivo - 2016.

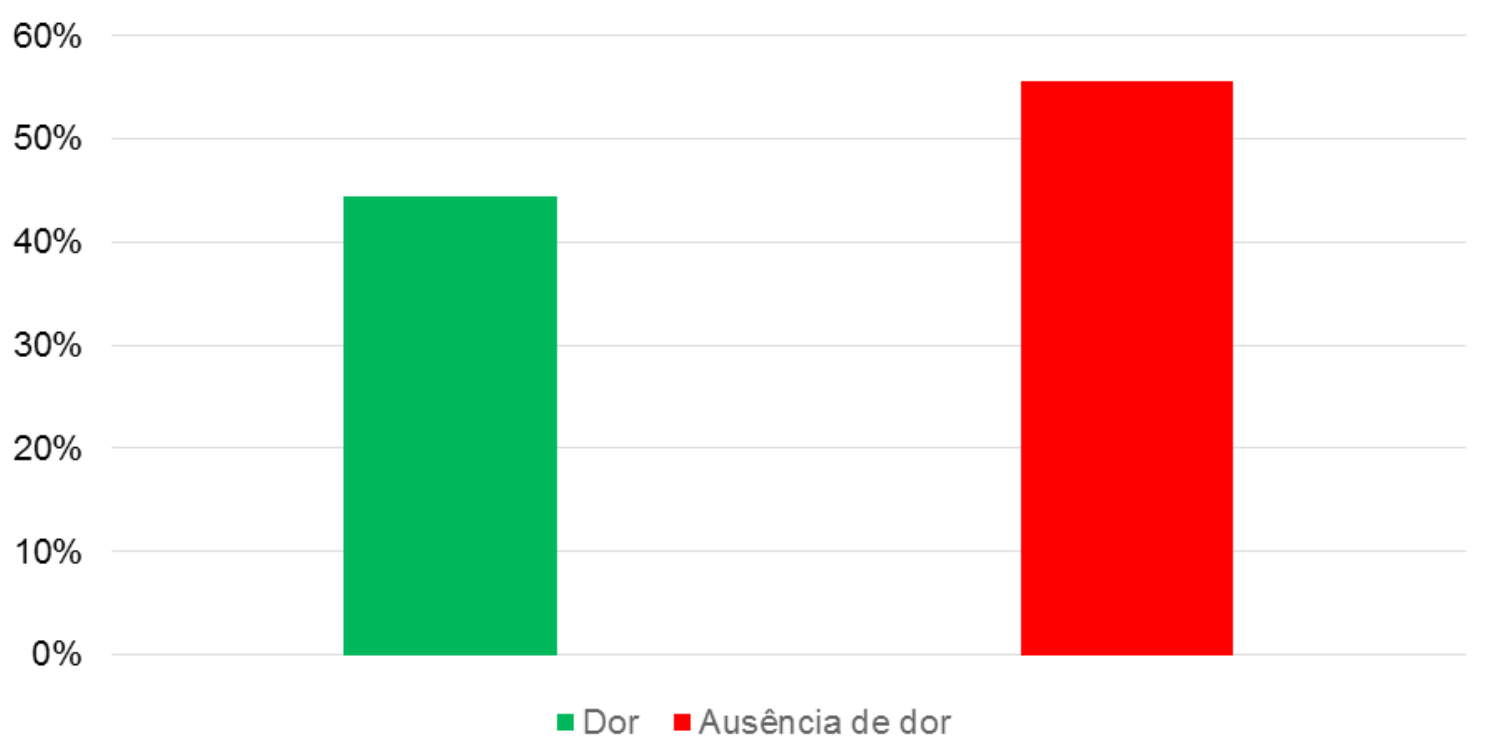

(clique para voltar ao texto) 